\title{
A multi-platform package for the analysis of intra- and interspecific trait evolution
}

\author{
Théo Gaboriau $^{1}$ (D) | Fábio K. Mendes ${ }^{2,3}$ | Simon Joly ${ }^{4,5}$ (D) | Daniele Silvestro ${ }^{6,7}$ | \\ Nicolas Salamin ${ }^{1}$
}

${ }^{1}$ Department of Computational Biology, University of Lausanne, Lausanne, Switzerland; ${ }^{2}$ School of Computer Science, The University of Auckland, Auckland, New Zealand; ${ }^{3}$ School of Biological Sciences, The University of Auckland, Auckland, New Zealand; ${ }^{4}$ Institut Recherche en Biologie Végétale, Montréal, QC, Canada; ${ }^{5}$ Montreal Botanical Garden, Montreal, QC, Canada; ${ }^{6}$ Department of Biology, University of Fribourg, Fribourg, Switzerland and ${ }^{7}$ Department of Biological and Environmental Sciences, University of Gothenburg and Global Gothenburg Biodiversity Centre, Gothenburg, Sweden

Correspondence

Théo Gaboriau

Email: theo.gaboriau@unil.ch

Funding information

Vetenskapsrådet, Grant/Award Number: 2015-04748; Stiftelsen för Strategisk Forskning; Schweizerischer Nationalfonds zur Förderung der Wissenschaftlichen Forschung, Grant/Award Number: 310030185223; Royal Marsden NHS Foundation Trust, Grant/Award Number: 16-UOA-277; Université de Lausanne

\begin{abstract}
1. Evolutionary forces affect the distribution of phenotypes both within and among species. Yet, at the macro-evolutionary scale, the evolution of intraspecific variance is rarely considered. Here, we present an $\mathrm{R}$ and a BEAST 2 implementation that extends the JIVE (Joint inter- and Intraspecific Variance Evolution) model aimed at the analysis of continuous trait evolution at both inter- and intraspecific level.

2. Using a hierarchical Bayesian approach, we implemented a range of models for continuous trait evolution that operate independently on species means and variances along a phylogeny. The package uses Markov chain Monte Carlo for the inference of parameters and the evaluation of model fit. JIVE is available in the BITE (Bayesian Integrative models of Trait Evolution) R package, as well as in BEAST 2. The two implementations offer the same continuous trait evolutionary models, but differ in their use and types of analyses. The $\mathrm{R}$ implementation allows for faster analyses by taking the phylogeny as data, while providing graphical and statistical functions as part of tools for model comparison, result parsing and summary, and plotting. In the BEAST 2 implementation, the species tree is a parameter, and both its topology and divergence times are jointly estimated with trait model parameters.

3. The BITE package and the BEAST 2 implementation introduce new frameworks within comparative phylogenetics that explicitly model intraspecific variance. These tools allow users to tackle long-standing questions in evolutionary biology, such as the identification of key evolutionary processes determining niche conservatism, niche partitioning, and life-history strategies.
\end{abstract}

KEYWORDS

Bayesian methods, bioinformatics, comparative analysis, evolutionary biology, software, statistics

Théo Gaboriau and Fábio K. Mendes contributed equally to this study. 


\section{1 | INTRODUCTION}

Estimating the tempo of phenotypic evolution is crucial to understanding the dynamics of trait divergence and adaptation. Several macroevolutionary models have been proposed to characterize the evolutionary processes that generate and maintain phenotypic and species diversity (Felsenstein, 1985). In the past decades, this class of models has bloomed to allow for the investigation of a variety of hypotheses (Pennell \& Harmon, 2013), such as the effects of environment-dependent (Clavel \& Morlon, 2017) or diversity-dependent (Drury, Clavel, Manceau, \& Morlon, 2016; Mahler, Revell, Glor, \& Losos, 2010) processes, the presence of selective regimes (Beaulieu, Jhwueng, Boettiger, \& O'Meara, 2012; Blomberg, Garland, \& Ives, 2003; Butler \& King, 2004; Hansen $\&$ Martins, 1996), as well as repeated patterns of change in the tempo of phenotypic evolution (Bokma, 2008; Duchen et al., 2017; Harmon, Weir, Brock, Glor, \& Challenger, 2008; Landis, Schraiber, \& Liang, 2013).

Most macroevolutionary models assume that the unit of evolution is the species and do not directly model intraspecific variation as a parameter in the study of phenotypic evolution. Instead, they often consider intraspecific variation as a variance-introducing factor to be dealt with by an error term (but see, Felsenstein, 2008; Ives, Midford, \& Garland, 2007; Mendes, Fuentes-González, Schraiber, \& Hahn, 2018; Revell \& Graham Reynolds, 2012; Silvestro, Kostikova, Litsios, Pearman, \& Salamin, 2015). Intraspecific variation is nonetheless not merely a nuisance parameter, but a key evolutionary component (Duchen, Alfaro, Rolland, Salamin, \& Silvestro, 2019). Many factors have been shown to affect the evolution of intraspecific variation, which in turn has an influence on the driving forces of species evolution and diversification (Darwin, 1859; MacArthur, 1965).

Empirical and theoretical studies clearly suggest that the evolution of intraspecific variation of a trait is informative of the underlying phenotypic evolutionary dynamics and their causes. Theoretical models demonstrate that intraspecific phenotypic variance is expected to increase with disruptive competition for resources in an empty niche space (Ackermann \& Doebeli, 2004; Aguilée, Gascuel, Lambert, \& Ferriere, 2018; Pontarp \& Petchey, 2018). In the presence of a new resource, Drosophila populations subject to more competition displayed a faster niche expansion towards the new resource associated with an increase of intraspecific phenotypic variance (Bolnick, 2001). Similarly, the colonization of a new territory can lead to an increase in the intraspecific variation in climatic niche (Broennimann et al., 2007; Urbanski et al., 2012). This effect, known as ecological release (Cox \& Ricklefs, 1977; Crowell, 1962), has also been observed at the macroevolutionary scale during events of island colonization (Duda \& Lee, 2009; Yoder et al., 2010) or adaptation to new environments (Des Roches, Robertson, Harmon, \& Rosenblum, 2011). The increase in intraspecific phenotypic variance is then expected to slow down and eventually reverse as phenotypic diversification and subsequent speciation events progressively fill niche space
(Ackermann \& Doebeli, 2004; Aguilée et al., 2018; Pontarp \& Petchey, 2018). This effect of interspecific competition has been shown to greatly reduce intraspecific variation in prey diversity, thus affecting phenotypic variation (Costa-Pereira, Araújo, Souza, \& Ingram, 2019; Schluter, 2000).

Several evolutionary forces are also expected to conserve intraspecific phenotypic variance at the macroevolutionary scale. Multiple studies have demonstrated that the effect of ecological release can be balanced by a selection-diversification trade-off allowing the niche position to shift while the niche width is maintained (Ackermann \& Doebeli, 2004; Aguilée et al., 2018; Good, Martis, \& Hallatschek, 2018; Sjodin, Ripa, \& Lundberg, 2018). Many life-history traits were also found to constrain intraspecific phenotypic variation such as individual generalization in three-spine sticklebacks (Bolnick et al., 2010), choice of breeding resources in burying beetles (Nicrophorinae; Hopwood, Moore, Tregenza, \& Royle, 2016) or life cycle in eriogonoids (Kostikova, Silvestro, Pearman, \& Salamin, 2016). Finally, strong selective pressures such as predation (Pontarp \& Petchey, 2018) or environmental instability (Fuentes \& Ferrada, 2017) are expected to limit niche expansion and thus constrain intraspecific phenotypic variation. Thus, exploring the dynamics of intraspecific phenotypic variation at the macroevolutionary scale by including it in the model could be both relevant due to its heritability, and particularly insightful to tackle long-standing evolutionary questions.

Out of a necessity for acknowledging intraspecific variance in evolutionary processes, the JIVE model (Joint Inter and intraspecific Variance Evolution; Kostikova et al., 2016) was developed as a hierarchical Bayesian framework to jointly estimate the evolutionary rates for both the means and variances of phenotypic traits. We present here a multi-platform implementation of the JIVE model to perform macroevolutionary analysis of both intra and interspecific phenotypic variation, based on species-level phylogenetic trees and individual observations. We release an $\mathrm{R}$ implementation of the JIVE model embedded in a new BITE package (Bayesian Integrative models for Trait Evolution; available at https://CRAN.R-proje ct.org/package=bite) and a complementary, entirely new BEAST 2 (Bouckaert, 2019) implementation (available at https://github.com/ fkmendes/contraband). Our implementations expand the original model by introducing more accurate model testing algorithms, a greater flexibility in the model configuration, and joint estimation of the underlying phylogenetic tree along with other parameters (BEAST 2 implementation).

\section{METHODS}

The JIVE model considers phenotypic means and variances as two independent random vectors, with each entry in the vectors representing the phenotypic mean and variance of a species, respectively (Figure 1). Phenotypic means and variances are assumed to evolve along the same species tree (and therefore expected to co-vary phylogenetically), but are otherwise independent of 


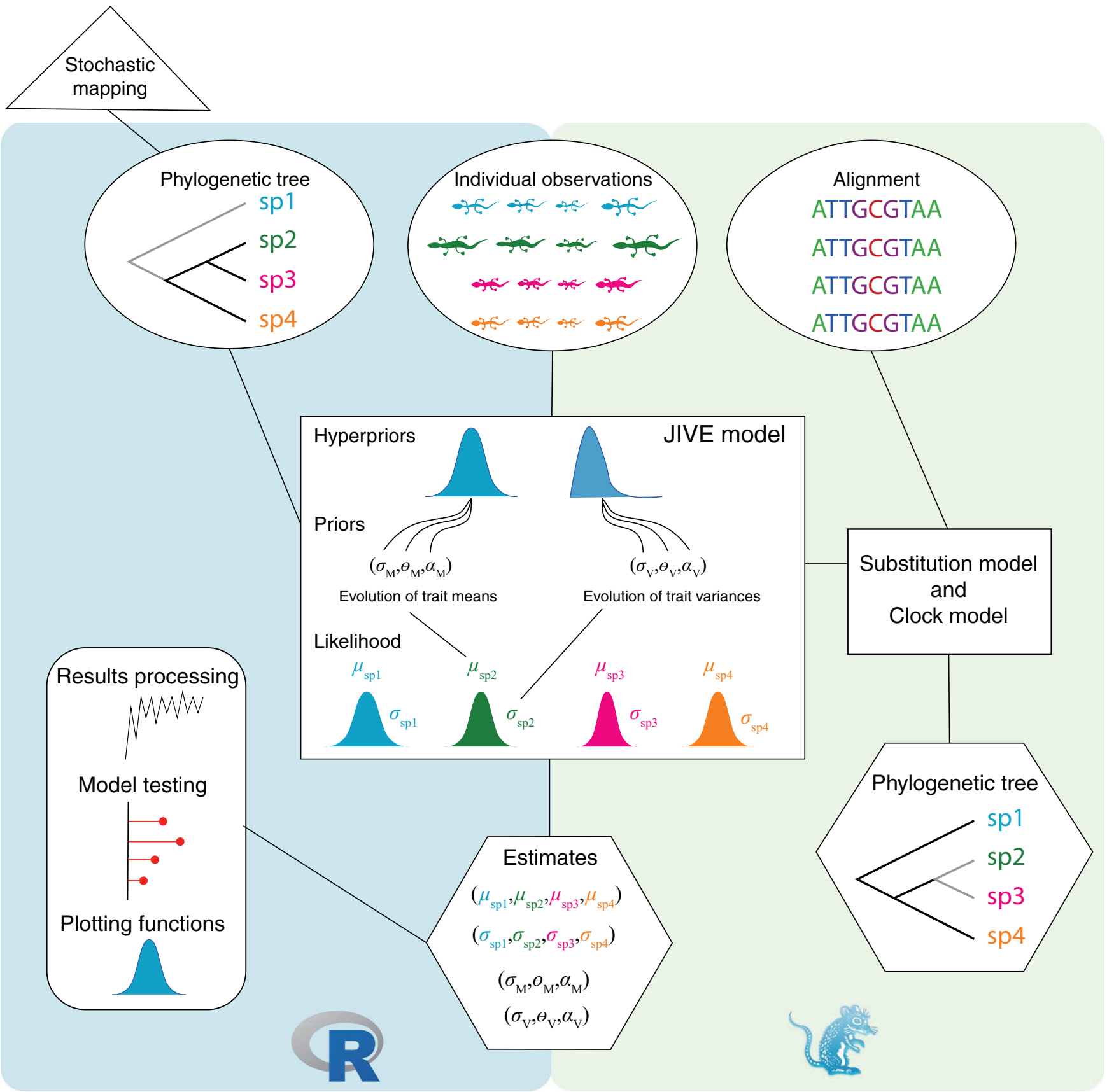

FIGURE 1 Overview of the Joint inter- and Intraspecific Variance Evolution (JIVE) implementations. Oval shapes represent data, rectangle boxes represent the specified models (prior distributions and likelihood functions) and hexagonal shapes represent parameter estimates after carrying out MCMC

each other and are not modelled as a bivariate normal distribution. A species' mean and variance then define a normal distribution, which is taken as the sampling distribution of trait values for multiple individuals of that species (i.e. for $n$ species, there will be $n$ normal distributions; Figure 1; while a normal distribution is likely a good model for intraspecific variation in a quantitative trait, technically any distribution could be used, as long as its probability density function can be computed. See the $\mathrm{R}$ package for an example). The JIVE model is thus more flexible than standard PCM approaches that assume traits are normally distributed within species. Currently, JIVE models multiple species phenotypic means and variances of a single continuous trait. Generalizing it to multiple continuous traits is possible in principle, but would require considerable increase in complexity and number of additional parameters.

JIVE is a hierarchical model, and as such can only be implemented in tools with support for flexible user-defined hierarchical model frameworks. To our knowledge, this feature is not readily available among popular maximum-likelihood packages, the majority of which are implemented in R (e.g, ouch, Butler \& King, 2004; phytools, phytools; geiger, Pennell et al., 2014; mvMORPH, Clavel, Escarguel, \& Merceron, 2015). The probability density function 
of the JIVE sampling distribution is considerably parameterized and complex, as opposed to non-hierarchical models conventionally used in comparative biology (e.g. a single multivariate normal distribution, when continuous trait evolution is modeled with Brownian motion; Felsenstein, 1985). Maximizing likelihood functions like JIVE is thus especially complicated by typical problems in maximum-likelihood estimation, such as convergence issues, the existence of local maxima, small samples biases, to name a few. Bayesian methods, on the other hand, circumvent some of these issues and provide a much more natural statistical framework for setting up hierarchical models (this is the approach we take; for more details, see below and the original work in Kostikova et al., 2016).

Finally, our modelling strategy differs markedly in nature from that of methods that incorporate 'measurement error' (Harmon \& Losos, 2005). Instead, it allows explicit testing of hypotheses about the evolution of intraspecific phenotypic variances (see below for an empirical example). These hypotheses can be informed by knowledge of how trait variation is expected to respond to or affect the characteristics of a species. Accounting for measurement error can decrease evolutionary rates estimates, for example, and increase estimates of phylogenetic signal (Ives et al., 2007)-such patterns are not expected under the JIVE model. Other methods consider 'measurement error' as the non-heritable component of variation in trait values (Lynch, 1991), also in stark contrast with JIVE.

Our complete rewrite of the original JIVE implementation (Kostikova et al., 2016) allowed us to significantly expand the flexibility of the model configuration. Furthermore, the new implementation achieved a substantial speedup (orders of magnitude), which makes the new JIVE model applicable to the large datasets available nowadays. We further extend the JIVE framework, generalizing the original $\mathrm{R}$ implementation so that it can be easily expanded to include other comparative models (to be used as the trait mean and variance priors, for example; see examples in the Supporting Information). Our general implementation and the hierarchical nature of JIVE also allow models other than the normal distribution for intraspecific variation (see examples in the Supporting Information). We provide new features such as model comparison methods, now based on faster and more accurate algorithms (stepping-stone sampling instead of thermodynamic integration, Xie, Lewis, Fan, Kuo, \& Chen, 2011), and utility functions with plotting and summary capabilities. Finally, the new $R$ version of the JIVE model improves on the original implementation by being more user-friendly and accessible through the CRAN repository.

The multi-platform release allows users to try alternative approaches depending on their familiarity with each platform, and the format and size of the data sets in hand (Figure 1). Our new implementation of JIVE in the BEAST 2 framework makes it possible to use other data such as molecular alignments and fossil occurrences together with continuous trait data in joint inferences of species trees and JIVE parameters. The JIVE model can be employed alongside a range of tree priors (e.g. Yule, birth-death, fossilized birth-death), substitution and clock models, and discrete morphological models. BEAST 2 also offers machinery for model comparison (e.g. stepping-stone sampling Xie et al., 2011). A long list of online tutorials and resources are available to the BEAST community (BaridoSottani et al., 2017).

We implemented different methods to allow for changes in evolutionary parameters across the branches of the tree. In the $\mathrm{R}$ version, where the phylogeny is assumed to be known, we map the evolutionary regimes along branches. These evolutionary regimes can be assigned based on pre-defined clades or obtained from the ancestral state inference of a discrete trait (e.g. stochastic mapping, Huelsenbeck, Nielsen, Bollback, \& Schultz, 2003; Revell, 2012a) that could have had an influence on the mode of evolution.

In the BEAST 2 implementation, the configuration of regimes across the nodes is sampled during the MCMC procedure, with a large number of regime shifts being penalized by a carefully selected prior so as to avoid unidentifiability issues. Our approach precludes the necessity of an a priori hypothesis on the specification of regimes, and is similar in nature to other Bayesian morphological clocks (e.g. Eastman, Alfaro, Joyce, Hipp, \& Harmon, 2011; Revell, Mahler, Peres-Neto, \& Redelings, 2011; Uyeda \& Harmon, 2014); it is however more general in that it allows for multiple regime shifts with homoplasy, while being readily used with both BM and OU models (i.e. different regimes can mean either evolutionary rates or adaptive optima). Sampling of shifts (which are assumed to happen at the root-end of branches) is done through Bayesian stochastic-search variable selection (George \& McCulloch, 1993) in a fashion analogous to that of the random local clock model already implemented in BEAST 2. More details about this can be found in the Supporting Information and in the original clock model reference (Drummond \& Suchard, 2010). Importantly, this is the first implementation (to our knowledge) of such a general morphological clock that can be used while accounting for phylogenetic uncertainty (in topology and divergence times) under a full probabilistic framework.

When choosing which implementation to use, we encourage the $\mathrm{R}$ version when the goal is to perform hypothesis testing and to compare alternative models, when the phylogeny is not particularly contentious (or when alignment data and fossil calibrations are not available), and for initial data exploration. Conversely, BEAST 2 analyses involving the JIVE model are recommended when novel data from multiple sources are available (and required models are not implemented in R), when no a priori hypotheses about evolutionary regime mappings have been proposed, or when there is evidence for rampant phylogenetic incongruenceinducing processes like incomplete lineage sorting (ILS; Mendes \& Hahn, 2016, 2018; Ogilvie, Bouckaert, \& Drummond, 2017). Finally, one of the strengths of our multi-platform release is that the user can easily set up the analysis in $\mathrm{R}$, carry it out using BEAST 2, and then read the log files and process it in $\mathrm{R}$ once again (Figure 1). 


\section{3 | DESCRIPTION}

The $\mathrm{R}$ package (BITE) containing the JIVE implementation is available from CRAN and includes example files to guide users in setting up analyses. In both $\mathrm{R}$ and BEAST 2, analyses with JIVE require at least two steps: model specification and parameter estimation. First, the user must create a variable we refer to as the 'JIVE object' that will contain all the information needed to run the model. This can be done by running the make_jive() function in $\mathrm{R}$ (Table 1). Here the user specifies the models for the evolution of means and variances and for intraspecific variation in trait values and the input data. Second, the user has the choice to run the JIVE model in R or in BEAST 2. In R, the mcmc_bite() function is called to run the inference procedure, based on the JIVE object previously created. To use the BEAST 2 implementation, the user has the option to use the $\mathrm{xml}$ _bite() function to prepare a BEAST 2 control file (an.xml file) from the same JIVE object. The BEAST 2 control file can then be used as in any analysis under this platform.

When running JIVE in $\mathrm{R}$ through bite prior to the second step, the user can modify several aspects of the MCMC algorithm using additional functions of the $\mathrm{R}$ package. The control_jive() function allows the modification of starting parameters value, window sizes for proposals, proposal methods and hyper-prior specification. The function hpfun() is used to define priors that can be parsed into

TAB LE 1 Overview of bite functions and their descriptions

\begin{tabular}{|c|c|}
\hline Function name & Description \\
\hline make_jive() & $\begin{array}{l}\text { Creates a JIVE object (template of a } \\
\text { hierarchical Bayesian PCM to be parsed into } \\
\text { mcmc bite()) }\end{array}$ \\
\hline control_jive() & Tunes MCMC paramaters in a JIVE object \\
\hline hpfun() & $\begin{array}{l}\text { creates a prior density function to be parsed } \\
\text { into } \mathrm{mcmc}_{\mathrm{B}} \text { bite() }\end{array}$ \\
\hline mcmc_bite() & $\begin{array}{l}\text { Runs MCMC algorithm according to the given } \\
\text { template }\end{array}$ \\
\hline marginal_lik() & Calculates marginal likelihood using output \\
\hline plot_bf() & $\begin{array}{l}\text { Plots summary of Bayes Factors (comparison } \\
\text { of marginal likelihoods) }\end{array}$ \\
\hline plot_hp() & $\begin{array}{l}\text { Plots prior density function from hpfun() or } \\
\text { make_jive() }\end{array}$ \\
\hline plot_jive() & $\begin{array}{l}\text { Plots input data and models from a JIVE } \\
\text { object }\end{array}$ \\
\hline plot_mcmc_bite() & $\begin{array}{l}\text { Plots traces and densities from a MCMC log } \\
\text { file }\end{array}$ \\
\hline plot_post_beast() & $\begin{array}{l}\text { Plots phylogenetic tree along with nodes } \\
\text { posterior probabilities from BEAST } 2\end{array}$ \\
\hline plot_pvo() & $\begin{array}{l}\text { Plots phylogenetic tree along with estimates } \\
\text { of phenotypic intraspecific variation }\end{array}$ \\
\hline sim_jive() & $\begin{array}{l}\text { Simulates trait evolution using the } \\
\text { hierarchical model }\end{array}$ \\
\hline xml_bite() & $\begin{array}{l}\text { Modifies.xml file from Beauti with JIVE } \\
\text { template }\end{array}$ \\
\hline
\end{tabular}

bite functions. These functions provide an easy and flexible way to tune the inference algorithm. The BITE package also implements algorithms for evaluating model fit and performing model comparison such as stepping-stone (Xie et al., 2011) and thermodynamic integration (TI, Lartillot, Philippe, \& Lewis, 2006). These approaches produce output files that can be parsed by the marginal_lik() function, which extracts the marginal likelihood of a model (i.e. the model evidence); then marginal likelihoods from different models can be compared using Bayes factor (Jeffreys, 1935; Kass \& Raftery, 1995).

Finally, the BITE R package presents a number of plotting functions to visually inspect and summarize model and hyper-prior specification (e.g. plot_jive(), Figure 2; plot_hp(), Figure S15), convergence of the $\mathrm{MCMC}$ chain, parameter posterior distributions (plot_mcmc_bite(), Figure S16), Bayes factor comparison (plot_bf(), Figure 3), and mean and variance estimates (plot_pvo(), Figure S18). Using these function, the user can therefore graphically explore both pre- and post-MCMC objects, from both the R and BEAST 2 implementations, within the $\mathrm{R}$ environment (e.g. Figure 5). Our BITE package has several R dependencies: APE (Paradis \& Schliep, 2019) and PHYTOOLs (Revell, 2012b) for phylogenetic trees handling, and coda (Plummer, Best, Cowles, \& Vines, 2006) for MCMC output analysis.

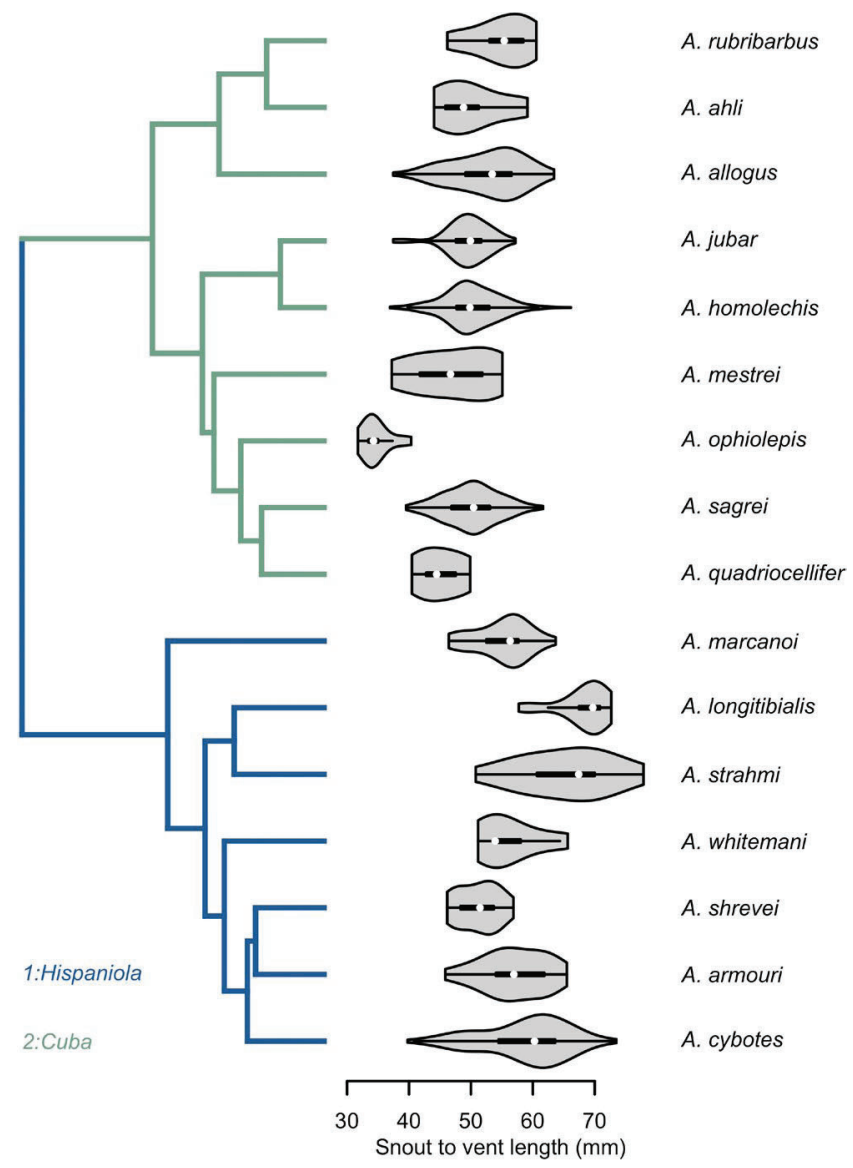

FIGURE 2 Output of the plot_jive() function displaying the Anolis data used as this example, as well as the assumed map of evolutionary regimes along the phylogeny 


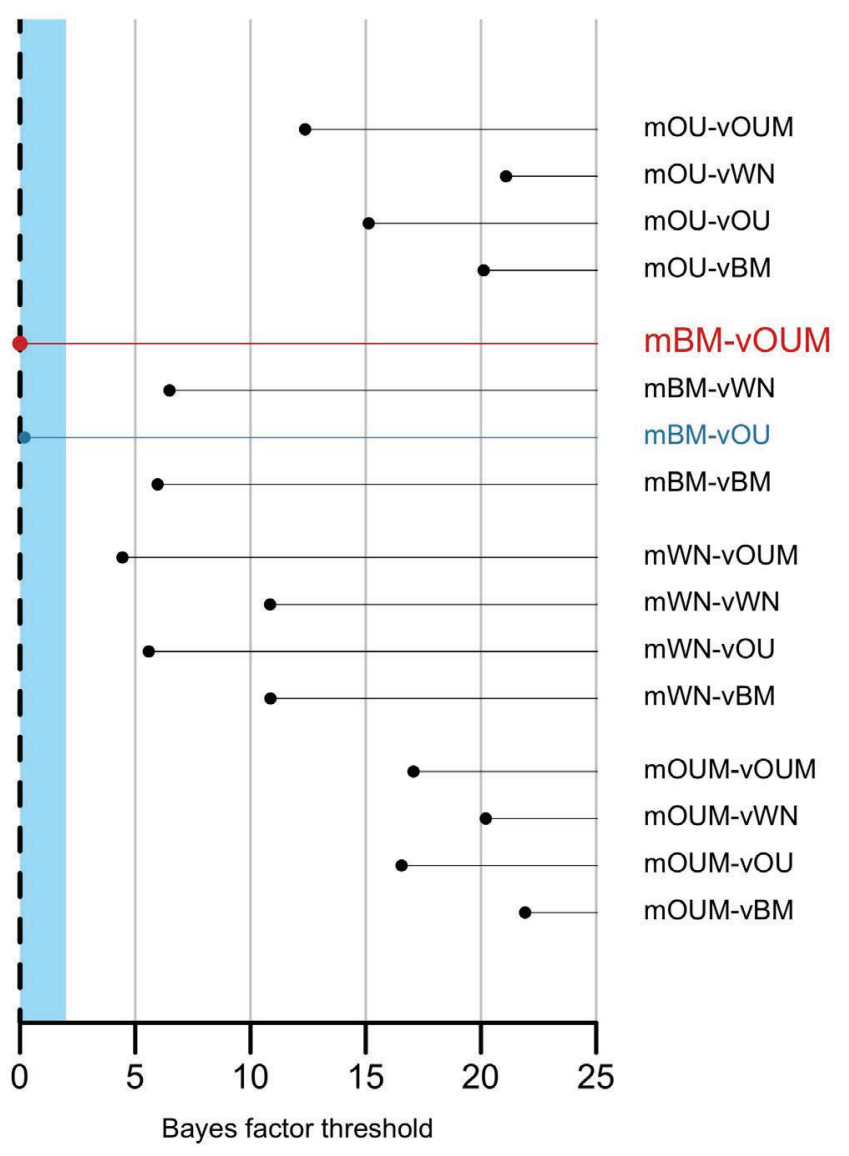

FIGURE 3 Output of the plot_bf() function displaying the Bayes Factors $\left(2\left(\log L_{T 1}\left(M_{1}\right)-\log L_{T 1}\left(M_{0}\right)\right)\right)$ between the model with the highest marginal likelihood and every other model. The area shaded in blue represents the 2 log unit-threshold under which the best model is not sufficiently supported. The 'lollipop' highlighted in red represents the best model $(B F=0)$

\section{4 | EXAMPLE: ELEVATION EFFECT ON THE INTRASPECIFIC VARIANCE OF BODY SIZE IN ANOLIS LIZARDS}

We illustrate the many new functionalities of our JIVE implementation by exploring the evolutionary history of body-size intraspecific variation during the Caribbean Anolis radiation. Caribbean Anolis lizards display large variation in body size resulting from convergent adaptation to different microhabitats within islands (Losos \& Ricklefs, 2009). This pattern suggests that deterministic ecological processes might be involved in diversification of body-size in Anolis. Further evidence can be found in the decreasing rates of body-size diversification as a function of the diversity of competing lineages, which points to a role of ecological release in the variation of body size within this clade (Mahler et al., 2010). Different patterns in bodysize distribution between clades inhabiting Cuba (sagrei; intraspecific altitudinal clines) and Hispaniola (cybotes; interspecific altitudinal clines) (Muñoz, Wegener, \& Algar, 2014) suggest that intraspecific variation might show different response to ecological opportunity. Differences in the physical geography of Cuba (small highland areas) and Hispaniola (large highland areas) may have triggered divergent evolutionary processes influencing both intra- and interspecific variance in body size (Muñoz et al., 2014).

Using JIVE, we tested alternative models of body-size evolution in Caribbean Anolis lizards, taking into account both inter- and intraspecific variation. We used a section of a previously estimated Anolis phylogeny from (Poe et al., 2017) and the dataset from (Muñoz et al., 2014) giving the snout-to-vent length (svl) of 1,106 individuals from 16 Anolis species (Figure 2) living in Cuba (sagrei clade) or Hispaniola (cybotes clade). Our goal is not to improve on the phylogenetic estimates from (Poe et al., 2017), but rather to showcase the new features and models of our implementations. Below, we present results from our $\mathrm{R}$ implementation, but also analyse the Anolis data set with our BEAST 2 implementation, briefly discussing some of its results (but see Supporting Information). Tutorials for both platforms accompany this manuscript (electronic Supporting Information, https://github.com/theogab/bite).

We tested four competing hypotheses for the evolution of both means and variances, leading to a combination of 16 alternative models. The first hypothesis (WN) assumes that either the variance or the mean of the continuous phenotypic trait (both henceforth referred to as the 'moments' of the trait) has no phylogenetic signal (i.e. body size moments are not heritable and completely determined by environmental gradients). The second hypothesis (BM) assumes that the phenotypic moment diffuses through the phylogeny with ever-increasing variance (i.e. body-size moments are inherited constrained by evolutionary forces). The third hypothesis (Ornstein-Uhlenbeck model, $\mathrm{OU}$ ) assumes that one adaptive optimum is shared by both clades and that the moments of body-size are constrained by selective pressures with no differences between islands. Finally, the fourth hypothesis (OUM) assumes different adaptive optima for Cuba and Hispaniola (i.e. the moments of body-size are constrained by selective pressures that differ between islands). For clarity, we referred to the models describing the evolution of species means with an ' $m$ ' prefix and to those describing the evolution of species variances with a ' $v$ ' prefix. If the mean was modelled with white noise and the variance with Brownian motion, we thus referred to this model as ' $m W N-v B M$ '.

We first ran independent MCMC chains of 2 million generations using $\mathrm{TI}$ sampling in order to compute the marginal likelihood of the 16 alternative model combinations to be used as the JIVE prior. The preferred prior model assumed that the species mean trait values evolved along the phylogeny according to a Brownian motion process $(\mathrm{mBM})$, and that the intraspecific variance evolved according to a multiple adaptive optima OU, with 'Cuba' and 'Hispaniola' having their own adaptive optimum (vOUM; Figure 3). Support for this model was strong against every other model except the mBM-vOU model (BF $<2$ ).

For the purpose of demonstration, we chose to continue the analysis with the more complex model, mBM-vOUM, even though it did not have a distinctively better fit compared to the second best model. We conducted parameter estimation with standard MCMC for 2 million generations (sampling every 1,000 generations, and discarding the first $10 \%$ as burn-in). We observed no significant difference in posterior estimates of $\theta$ for the 'Hispaniola' and 'Cuba' clades, with the former exhibiting slightly larger trait variances (Figure 4), 


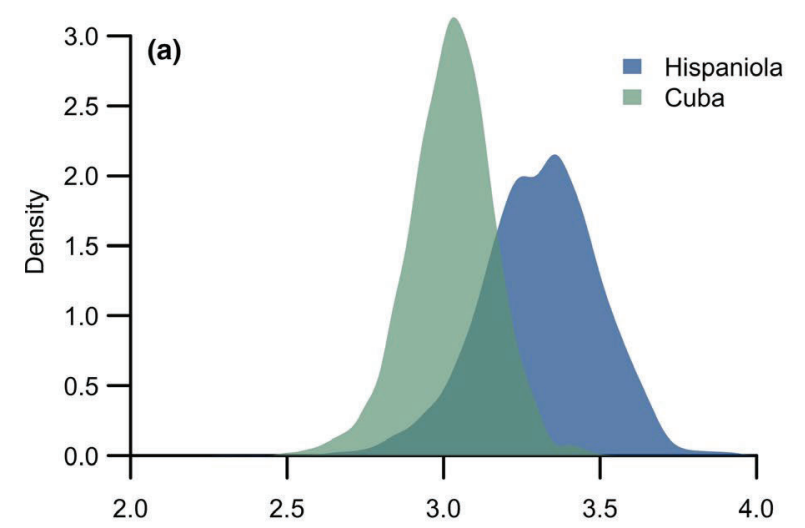

$\theta_{\mathrm{H}}$ and $\theta_{\mathrm{C}}$ (log-variance) posterior estimates for snout to vent length

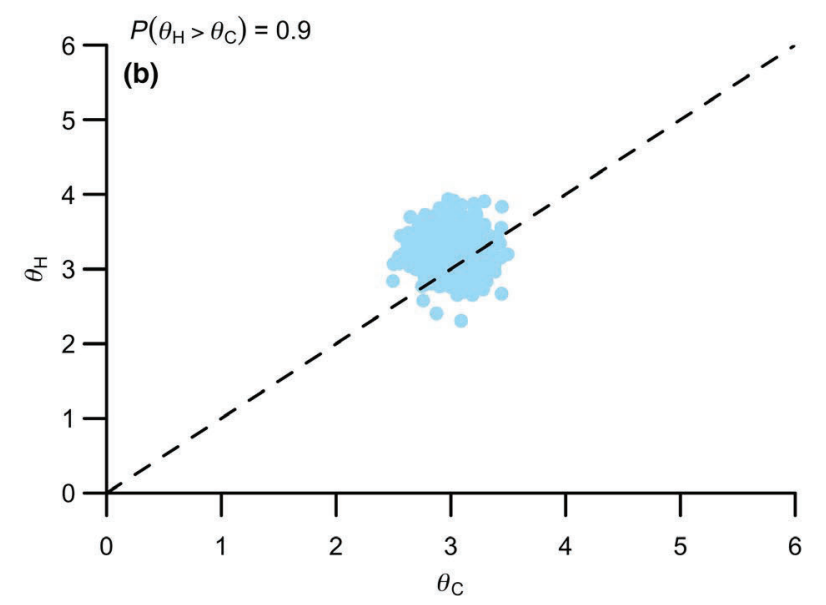

FIGURE 4 (a) Posterior density curves of the estimated optima for 'cybotes' ( $\theta_{\mathrm{H}}$, blue density curve) and 'sagrei' $\left(\theta_{\mathrm{C}}\right.$, green density curve) clades under the best model (mBM-vOUM). (b) Posterior estimates of the optimal variance for 'cybotes' $\left(\theta_{H}\right)$ versus 'sagrei' $\left(\theta_{\mathrm{C}}\right)$ clades under the best model (mBM-vOUM). We see that there is no clear support for $\theta_{\mathrm{H}}>\theta_{\mathrm{C}}$

which was expected given the small Bayes factor between the mBM-vOU and mBM-vOUM models. We also estimated JIVE and prior parameters using BEAST 2 under the mBM-vOUM model, using molecular data from (Poe et al., 2017) to infer the phylogeny and the mapping of evolutionary regimes (see Supporting Information). Unsurprisingly, we recovered the same tree as that in (Poe et al., 2017; Figure 5). We observed higher mean $\theta$ values within 'Hispaniola' compared to 'Cuba'. Furthermore, species with low observed trait variances (i.e. calculated from their trait value samples), such as $A$. ophiolepis, and very high trait variances, such as $A$. cybotes and A. strahmi, were estimated to have low and high $\theta$ values, accordingly. Together, these results show that strong evolutionary forces constrained the intraspecific variation of body size in Anolis lizards, while the evolution of their mean body size seems less constrained. Two potential issues should be mentioned regarding this analysis. First, the island effect is informed by a single data point, which limits the statistical power of the study (Maddison \& Fitzjohn, 2015). Second, the differences between islands are not sufficiently pronounced to draw bolder conclusions. Despite these issues, we note that parameter estimates contrast with

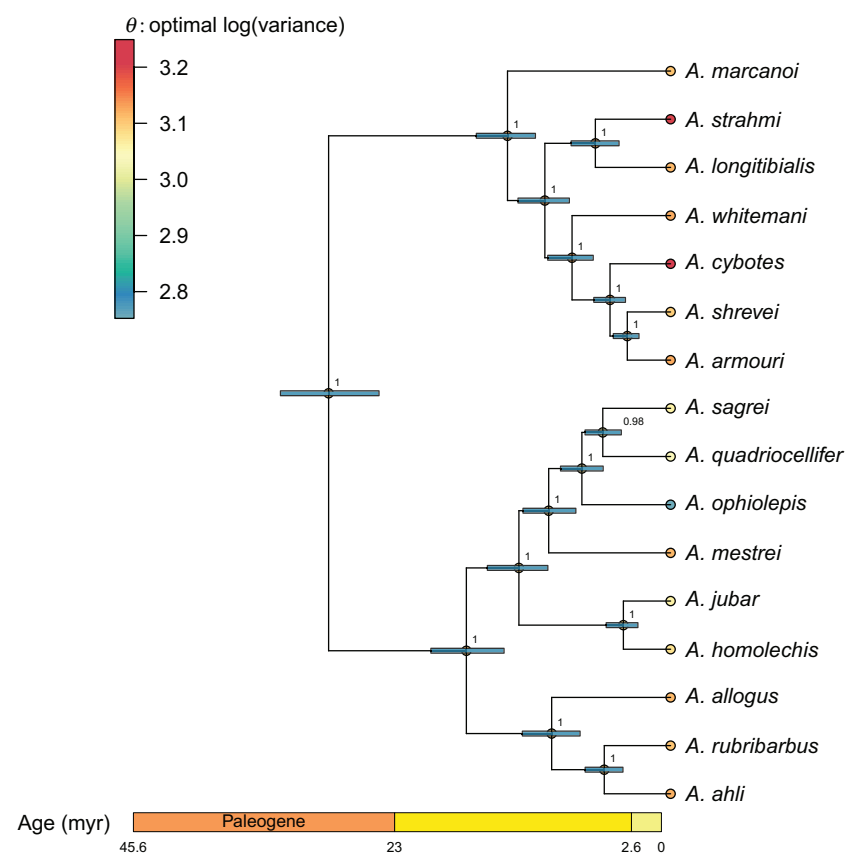

FIGURE 5 Maximum-credibility Anolis tree estimated from molecular and inter and intraspecific continuous trait data (using BEAST 2). Nodes are colored according to trait variance mean posterior probabilities of $\theta$, under the vOUM model. Bars correspond to $95 \%$ highest posterior density intervals for divergence times, and numbers next to nodes to posterior clade probabilities

the observed inverse Bergman's clines in this clade (Muñoz et al., 2014), where an interspecific cline in body size was identified in Hispaniola while an intraspecific cline was identified in Cuba. Here, the steep topography of Hispaniola might have reduced exchanges between populations and subsequently competition. Ecological release theory posits that there are several phases to adaptive radiation (Ackermann \& Doebeli, 2004; Aguilée et al., 2018; Pontarp \& Petchey, 2018). A first phase, dominated by disruptive competition, increases intraspecific variation (Bolnick, 2001), while a second phase, dominated by interspecific density effects, triggers specialization and decreases intraspecific variation. The steep topography of Hispaniola may have reduced the influence of interspecific density effects as opposed to Cuba, where the less fragmented topography might have triggered competition between Anolis species, inducing niche partitioning. Thus, divergence on intraspecific variation between sagrei and cybotes clades might come from the different stages of adaptive radiation they are facing due topographic variation between islands.

\section{ACKNOWLEDGEMENTS}

F.K.M. was supported by Marsden grant 16-UOA-277. D.S. received funding from the Swiss National Science Foundation (PCEFP3_187012; FN-1749) and from the Swedish Research Council (VR: 2019-04739). N.S. received funding from the Swiss National Science Foundation (310030-185223) and from the University of Lausanne. We thank the computing infrastructure of the University of Lausanne for access to computing clusters. 


\section{AUTHORS' CONTRIBUTIONS}

T.G. coded the R package with contributions from S.J., F.K.M. coded the Beast 2 implementation; T.G. and F.K.M. performed the analyses; T.G., F.K.M., D.S. and N.S. wrote the manuscript; D.S and N.S. coordinated the study.

\section{PEER REVIEW}

The peer review history for this article is available at https://publons. com/publon/10.1111/2041-210X.13458.

\section{DATA AVAILABILITY STATEMENT}

Data used in this manuscript can be found in the Dryad Digital Repository https://doi.org/10.5061/dryad.q39h2 (Muñoz et al., 2014).

\section{ORCID}

Théo Gaboriau (D) https://orcid.org/0000-0001-7530-2204

Simon Joly iD https://orcid.org/0000-0002-0865-8267

\section{REFERENCES}

Ackermann, M., \& Doebeli, M. (2004). Evolution of niche width and adaptive diversification. Evolution, 58(12), 2599-2612. https://doi. org/10.1554/04-244

Aguilée, R., Gascuel, F., Lambert, A., \& Ferriere, R. (2018). Clade diversification dynamics and the biotic and abiotic controls of speciation and extinction rates. Nature Communications, 9(1), 3013. https://doi. org/10.1038/s41467-018-05419-7

Barido-Sottani, J., Bošková, V., Du Plessis, L., Kühnert, D., Carsten, M., Mitov, V., ... Stadler, T. (2017). Taming the BEAST - A community teaching material resource for BEAST 2. Systematic Biology, 67, 170174. https://doi.org/10.1093/sysbio/syx060

Beaulieu, J. M., Jhwueng, D.-C., Boettiger, C., \& O'Meara, B. C. (2012). Modeling stabilizing selection: Expanding the Ornstein-Uhlenbeck model of adaptive evolution. Evolution, 66(8), 2369-2383. https:// doi.org/10.1111/j.1558-5646.2012.01619.x

Blomberg, S. P., Garland Jr., T., \& Ives, A. R. (2003). Signal in comparative data: Testing for phylogenetic behavioral traits are more labile. Evolution, 57(4), 717-745.

Bokma, F. (2008). Detection of 'punctuated equilibrium' by Bayesian estimation of speciation and extinction rates, ancestral character states, and rates of anagenetic and cladogenetic evolution on a molecular phylogeny. Evolution, 62(11), 2718-2726. https://doi.org/10.1111/j.1558-5646.2008.00492.x

Bolnick, D. I. (2001). Intraspecific competition favours niche width expansion in Drosophila melanogaster. Nature, 4, 463-466. https://doi. org/10.1038/35068555

Bolnick, D. I., Ingram, T., Stutz, W. E., Snowberg, L. K., Lau, O. L., \& Paull, J. S. (2010). Ecological release from interspecific competition leads to decoupled changes in population and individual niche width. Proceedings of the Royal Society B: Biological Sciences, 277(1689), 1789-1797. https://doi.org/10.1098/rspb.2010.0018

Bouckaert, R., Vaughan, T. G., Barido-Sottani, J., Duchêne, S., Fourment, M., Gavryushkina, A., ... Matschiner, M. (2019). BEAST 2.5: An advanced software platform for Bayesian evolutionary analysis. PLoS Computational Biology, 15, e1006650.

Broennimann, O., Treier, U. A., Müller-Schärer, H., Thuiller, W., Peterson, A. T., \& Guisan, A. (2007). Evidence of climatic niche shift during biological invasion. Ecology Letters, 10(8), 701-709. https://doi. org/10.1111/j.1461-0248.2007.01060.x

Butler, M. A., \& King, A. A. (2004). Phylogenetic comparative analysis: A modeling approach for adaptive evolution. The American Naturalist, 164(6), 683-695. https://doi.org/10.1086/426002
Clavel, J., Escarguel, G., \& Merceron, G. (2015). mvMORPH: An R package for fitting multivariate evolutionary models to morphometric data. Methods in Ecology and Evolution, 6, 1311-1319. https://doi. org/10.1111/2041-210x.12420

Clavel, J., \& Morlon, H. (2017). Accelerated body size evolution during cold climatic periods in the Cenozoic. Proceedings of the National Academy of Sciences of the United States of America, 114(16), 41834188. https://doi.org/10.1073/pnas.1606868114

Costa-Pereira, R., Araújo, M. S., Souza, F. L., \& Ingram, T. (2019). Competition and resource breadth shape niche variation and overlap in multiple trophic dimensions. Proceedings of the Royal Society B: Biological Sciences, 286(1902). https://doi.org/10.1098/rspb.2019.0369

Cox, G. W., \& Ricklefs, R. E. (1977). Species diversity and ecological release in Caribbean land bird faunas. Oikos, 28(1), 113-122.

Crowell, K. L. (1962). Reduced interspecific competition among the birds of Bermuda. Ecology, 43(1), 75-88. https://doi.org/10.2307/1932042

Darwin, C. (1859). On the origin of species by means of natural selection, or, the preservation of favoured races in the struggle for life. London, UK: John Murray.

Des Roches, S., Robertson, J. M., Harmon, L. J., \& Rosenblum, E. B. (2011). Ecological release in White Sands lizards. Ecology and Evolution, 1(4), 571-578. https://doi.org/10.1002/ece3.50

Drummond, A. J., \& Suchard, M. A. (2010). Bayesian random local clocks, or one rate to rule them all.BMCBiology, 8. https://doi.org/10.1186/17417007-8-114

Drury, J., Clavel, J., Manceau, M., \& Morlon, H. (2016). Estimating the effect of competition on trait evolution using maximum likelihood inference. Systematic Biology, 65(4), 700-710. https://doi.org/10.1093/ sysbio/syw020

Duchen, P., Alfaro, M. L., Rolland, J., Salamin, N., \& Silvestro, D. (2019). On the effect of asymmetrical trait inheritance on models of trait evolution. bioRxiv, 768820

Duchen, P., Leuenberger, C., Szilágyi, S. M., Harmon, L., Eastman, J., Schweizer, M., \& Wegmann, D. (2017). Inference of evolutionary jumps in large phylogenies using Lévy processes. Systematic Biology, 66(6), 950-963. https://doi.org/10.1093/sysbio/syx028

Duda, T. F. J., \& Lee, T. (2009). Ecological release and venom evolution of a predatory marine snail at Easter Island. PLoS ONE, 4(5), 1-7.

Eastman, J. M., Alfaro, M. E., Joyce, P., Hipp, A. L., \& Harmon, L. J. (2011). A novel comparative method for identifying shifts in the rate of character evolution on trees. Evolution, 65, 3578-3589. https://doi. org/10.1111/j.1558-5646.2011.01401.x

Felsenstein, J. (1985). Phylogenies and the comparative method. The American Naturalist, 125(1), 1-15.

Felsenstein, J. (2008). Comparative methods with sampling error and within-species variation: Contrasts revisited and revised. The American Naturalist, 171(6), 713-725. https://doi.org/10.1086/587525

Fuentes, M. A., \& Ferrada, E. (2017). Environmental fluctuations and their consequences for the evolution of phenotypic diversity. Frontiers in Physics, 5, 1-7. https://doi.org/10.3389/fphy.2017.00016

George, E. I., \& McCulloch, R. E. (1993). Variable selection via Gibbs sampling. Journal of the American Statistical Association, 88, 881-889. https://doi.org/10.1080/01621459.1993.10476353

Good, B. H., Martis, S., \& Hallatschek, O. (2018). Adaptation limits ecological diversification and promotes ecological tinkering during the competition for substitutable resources. Proceedings of the National Academy of Sciences of the United States of America, 115(44), E10407-E10416. https://doi.org/10.1073/pnas.1807530115

Hansen, T. F., \& Martins, E. P. (1996). Translating between microevolutionary process and macroevolutionary patterns: The correlation structure of interspecific data. Evolution, 50(4), 1404-1417. https:// doi.org/10.2307/2410878

Harmon, L. J., \& Losos, J. B. (2005). The effect of intraspecific sample size on type I and type II error rates in comparative studies. Evolution, 59(12), 2705-2710. https://doi.org/10.1554/05-224.1 
Harmon, L. J., Weir, J. T., Brock, C. D., Glor, R. E., \& Challenger, W. (2008). GEIGER: Investigating evolutionary radiations. Bioinformatics, 24(1), 129-131. https://doi.org/10.1093/bioinformatics/btm538

Hopwood, P. E., Moore, A. J., Tregenza, T., \& Royle, N. J. (2016). Niche variation and the maintenance of variation. Ecological Entomology, 41, 96-104. https://doi.org/10.1111/een.12275

Huelsenbeck, J. P., Nielsen, R., Bollback, J. P., \& Schultz, T. (2003). Stochastic mapping of morphological characters. Systematic Biology, 52(2), 131-158. https://doi.org/10.1080/10635150390192780

Ives, A. R., Midford, P. E., \& Garland, T. (2007). Within-species variation and measurement error in phylogenetic comparative methods. Systematic Biology, 56(2), 252-270. https://doi.org/10.1080/10635 150701313830

Jeffreys, H. (1935). Some tests of significance, treated by the theory of probability. Mathematical Proceedings of the Cambridge Philosophical Society, 31(2), 203-222. https://doi.org/10.1017/s030500410001330x

Kass, R. E., \& Raftery, A. E. (1995). Bayes factors and model uncertainty. Journal of the American Statistical Association, 90(43), 773-795.

Kostikova, A., Silvestro, D., Pearman, P. B., \& Salamin, N. (2016). Bridging inter- and intraspecific trait evolution with a hierarchical Bayesian approach. Systematic Biology, 65(3), 417-431. https://doi.org/10.1093/ sysbio/syw010

Landis, M. J., Schraiber, J. G., \& Liang, M. (2013). Phylogenetic analysis using Lévy processes: Finding jumps in the evolution of continuous traits. Systematic Biology, 62(2), 193-204. https://doi.org/10.1093/ sysbio/sys086

Lartillot, N., Philippe, H., \& Lewis, P. (2006). Computing Bayes factors using thermodynamic integration. Systematic Biology, 55(2), 195-207. https://doi.org/10.1080/10635150500433722

Losos, J. B., \& Ricklefs, R. E. (2009). Adaptation and diversification on islands. Nature, 457, 830-836. https://doi.org/10.1038/nature07893

Lynch, M. (1991). Methods for the analysis of comparative data in evolutionary biology. Evolution, 45, 1065-1080. https://doi.org/10.2307/ 2409716

MacArthur, R. (1965). Patterns of species diversity. Biological Reviews of the Cambridge Philosophical Society, 40(4), 510-533.

Maddison, W. P., \& Fitzjohn, R. G. (2015). The unsolved challenge to phylogenetic correlation tests for categorical characters. Systematic Biology, 64(1), 127-136. https://doi.org/10.1093/sysbio/syu070

Mahler, D. L., Revell, L. J., Glor, R. E., \& Losos, J. B. (2010). Ecological opportunity and the rate of morphological evolution in the diversification of greater Antillean anoles. Evolution, 64(9), 2731-2745. https:// doi.org/10.1111/j.1558-5646.2010.01026.x

Mendes, F. K., Fuentes-González, J. A., Schraiber, J., \& Hahn, M. W. (2018). A multispecies coalescent model for quantitative traits. eLife, e364482.

Mendes, F. K., \& Hahn, M. W. (2016). Gene tree discordance can cause apparent substitution rate variation. Systematic Biology, 65, 711-721. https://doi.org/10.1093/sysbio/syw018

Mendes, F. K., \& Hahn, M. W. (2018). Why concatenation fails near the anomaly zone. Systematic Biology, 67, 158-169. https://doi. org/10.1093/sysbio/syx063

Muñoz, M. M., Wegener, J. E., \& Algar, A. C. (2014). Data from: Untangling intra- and interspecific effects on body size clines reveals divergent processes structuring convergent patterns in Anolis lizards. Dryad Digital Repository, https://doi.org/10.5061/dryad.q39h2

Ogilvie, H. A., Bouckaert, R. R., \& Drummond, A. J. (2017). StarBEAST2 brings faster species tree inference and accurate estimates of substitution rates. Molecular Biology and Evolution, 34, 2101-2114. https:// doi.org/10.1093/molbev/msx126

Paradis, E., \& Schliep, K. (2019). ape 5.0: An environment for modern phylogenetics and evolutionary analyses in R. Bioinformatics, 35(3), 526-528. https://doi.org/10.1093/bioinformatics/bty633

Pennell, M. W., Eastman, J. M., Slater, G. J., Brown, J. W., Uyeda, J. C., FitzJohn, R. G., ... Harmon, L. J. (2014). geiger v2.0: An expanded suite of methods for fitting macroevolutionary models to phylogenetic trees. Bioinformatics, 30, 2216-2218. https://doi.org/10.1093/bioinformatics/btu181

Pennell, M. W., \& Harmon, L. J. (2013). An integrative view of phylogenetic comparative methods: Connections to population genetics, community ecology, and paleobiology. Annals of the New York Academy of Sciences, 1289(1), 90-105. https://doi.org/10.3410/f.718022005.793479088

Plummer, M., Best, N., Cowles, K., \& Vines, K. (2006). CODA: Convergence diagnosis and output analysis for MCMC. $R$ News, 6(1), 7-11.

Poe, S., Nieto-Montes de Oca, A., Torres-carvajal, O., De Queiroz, K., Velasco, J. A., Truett, B., ... Latella, I. (2017). A phylogenetic, biogeographic, and taxonomic study of all extant species of Anolis (Squamata; Iguanidae). Systematic Biology, 66(5), 663-697. https:// doi.org/10.1093/sysbio/syx029

Pontarp, M., \& Petchey, O. L. (2018). Ecological opportunity and predator-prey interactions: Linking eco-evolutionary processes and diversification in adaptive radiations. Proceedings of the Royal Society B: Biological Sciences, 285(1874). https://doi.org/10.1098/rspb.2017.2550

Revell, L. J. (2012). phytools: An R package for phylogenetic comparative biology (and other things). Methods in Ecology and Evolution, 3, 217-223. https://doi.org/10.1111/j.2041-210X.2011.00169.x

Revell, L. J., \& Graham Reynolds, R. (2012). A new Bayesian method for fitting evolutionary models to comparative data with intraspecific variation. Evolution, 66(9), 2697-2707. https://doi. org/10.1111/j.1558-5646.2012.01645.x

Revell, L. J., Mahler, D. L., Peres-Neto, P. R., \& Redelings, B. D. (2011). A new phylogenetic method for identifying exceptional phenotypic diversification. Evolution, 66, 135-146. https://doi.org/10.1111/j.15585646.2011.01435.x

Schluter, D. (2000). The ecology of adaptive radiation. New York, NY: Oxford University Press.

Silvestro, D., Kostikova, A., Litsios, G., Pearman, P. B., \& Salamin, N. (2015). Measurement errors should always be incorporated in phylogenetic comparative analysis. Methods in Ecology and Evolution, 6(3), 340-346.

Sjödin, H., Ripa, J., \& Lundberg, P. (2018). Principles of niche expansion. Proceedings of the Royal Society B: Biological Sciences, 285(1893). https://doi.org/10.1098/rspb.2018.2603

Urbanski, J., Mogi, M., O'Donnell, D., DeCotiis, M., Toma, T., \& Armbruster, P. (2012). Rapid adaptive evolution of photoperiodic response during invasion and range expansion across a climatic gradient. The American Naturalist, 179(4), 490-500. https://doi.org/10.1086/664709

Uyeda, J. C., \& Harmon, L. J. (2014). A novel Bayesian method for inferring and interpreting the dynamics of adaptive landscapes from phylogenetic comparative data. Systematic Biology, 63(6), 902-918. https://doi.org/10.1093/sysbio/syu057

Xie, W., Lewis, P. O., Fan, Y., Kuo, L., \& Chen, M.-H. (2011). Improving marginal likelihood estimation for Bayesian phylogenetic model selection. Systematic Biology, 60(2), 150-160. https://doi.org/10.1093/sysbio/syq085

Yoder, J. B., Clancey, E., Roches, S. D. E. S., Eastman, J. M., Gentry, L., Godsoe, W., ... Harmon, L. J. (2010). Ecological opportunity and the origin of adaptive radiations. Journal of Evolutionary Biology, 23, 1581-1596. https://doi.org/10.1111/j.1420-9101.2010.02029.x

\section{SUPPORTING INFORMATION}

Additional supporting information may be found online in the Supporting Information section. 